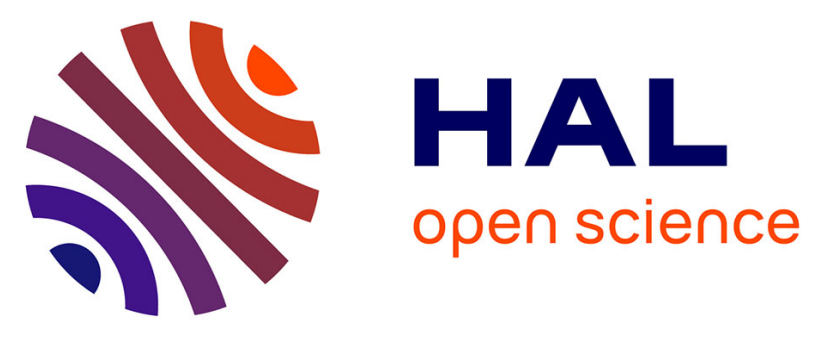

\title{
Differentiating between axonal damage and demyelination in healthy aging by combining diffusion-tensor imaging and diffusion-weighted spectroscopy in the human corpus callosum at $7 \mathrm{~T}$
} Francesca Branzoli, Ece Ercan, Romain Valabrègue, Emily T. Wood, Mathijs Buijs, Andrew Webb, Itamar Ronen

\section{To cite this version:}

Francesca Branzoli, Ece Ercan, Romain Valabrègue, Emily T. Wood, Mathijs Buijs, et al.. Differentiating between axonal damage and demyelination in healthy aging by combining diffusion-tensor imaging and diffusion-weighted spectroscopy in the human corpus callosum at $7 \mathrm{~T}$. Neurobiology of Aging, 2016, 47, pp.210-217. 10.1016/j.neurobiolaging.2016.07.022 . hal-01376793

\section{HAL Id: hal-01376793 \\ https://hal.sorbonne-universite.fr/hal-01376793}

Submitted on 5 Oct 2016

HAL is a multi-disciplinary open access archive for the deposit and dissemination of scientific research documents, whether they are published or not. The documents may come from teaching and research institutions in France or abroad, or from public or private research centers.
L'archive ouverte pluridisciplinaire HAL, est destinée au dépôt et à la diffusion de documents scientifiques de niveau recherche, publiés ou non, émanant des établissements d'enseignement et de recherche français ou étrangers, des laboratoires publics ou privés. 
Title: Differentiating between axonal damage and demyelination in healthy aging by combining diffusion-tensor imaging and diffusion-weighted spectroscopy in the human corpus callosum at $7 \mathrm{~T}$

Authors: ${ }^{a, b}$ Francesca Branzoli, ${ }^{c}$ Ece Ercan, ${ }^{a, b}$ Romain Valabrègue, ${ }^{d}$ Emily T. Wood, ${ }^{c}$ M. Buijs, ${ }^{c}$ A. Webb, and ${ }^{\mathrm{c}} \mathrm{I}$. Ronen

${ }^{a}$ Centre de NeuroImagerie de Recherche - CENIR, Institut du Cerveau et de la Moelle épinière ICM, Paris, France

'borbonne Universités, UPMC Paris 06, Inserm UMR S 1127, CNRS UMR 7225, F-75013, Paris, France

${ }^{\mathrm{c}}$ C. J. Gorter Center for High Field MRI, Department of Radiology, Leiden University Medical Center, Leiden, Netherlands

${ }^{\mathrm{d}}$ NeuroImmunology Branch (NINDS), National Institutes of Health, Bethesda, MD, United States

Email addresses:

eceercan@gmail.com

romain.valabregue@upmc.fr

emilytwood@gmail.com

m.buijs@lumc.nl

a.webb@lumc.nl

i.ronen@lumc.nl

\section{Corresponding author:}

Francesca Branzoli

CENIR - Centre de Neuroimagerie de Recherche

Bâtiment ICM

47 boulevard de l'Hôpital

75013 Paris

email: f.branzoli-ihu@icm-institute.org 


\begin{abstract}
Diffusion tensor imaging (DTI) and single voxel diffusion-weighted spectroscopy (DW-MRS) were used at $7 \mathrm{~T}$ to explore in vivo age-related microstructural changes in the corpus callosum (CC). Sixteen healthy elderly (age range 60-71 years) and thirteen healthy younger controls (age range 2332 years) were included in the study. In healthy elderly, we found lower water fractional anisotropy and higher water mean diffusivity and radial diffusivity in the $\mathrm{CC}$, indicating the onset of demyelination processes with healthy aging. These changes were not associated with a concomitant significant difference in the cytosolic diffusivity of the intra-axonal metabolite $\mathrm{N}$-acetylaspartate ( $\mathrm{p}$ $=0.12$ ), the latter representing a pure measure of intra-axonal integrity. It was concluded that the possible intra-axonal changes associated with normal aging processes are below the detection level of DW-MRS in our experiment (e.g. smaller than 10\%) in the age range investigated. Lower axial diffusivity of total creatine was observed in the elderly group $(\mathrm{p}=0.058)$, possibly linked to a dysfunction in the energy metabolism associated with a deficit in myelin synthesis.
\end{abstract}

Keywords: diffusion-weighted MRS, intracellular metabolites, DTI, healthy aging, white matter 


\section{Introduction}

Diffusion tensor imaging (DTI) has provided much evidence of age-related changes in human brain white matter (WM) microstructure, see e.g. (Salat, et al., 2005,Sullivan and Pfefferbaum, 2006). Lower fractional anisotropy (FA) and higher water mean diffusivity (MD) have been consistently detected in several white matter regions, suggesting alteration of WM microstructure in healthy aging, even when concomitant volume declines are not detectable (Coutu, et al., 2014,Madden, et al., 2012,Sullivan and Pfefferbaum, 2006). DTI analysis has suggested that the primary neurobiological mechanism associated with age-related degeneration in WM is a decline in the structural integrity of myelin (Wozniak and Lim, 2006). This hypothesis was corroborated by histological analyses performed in the rhesus monkey as well as in human post-mortem samples, showing that age-related alterations in the structure of myelin sheaths precede the loss of myelinated nerve fibers (Murray, et al., 2012,Peters, 2002,Peters and Kemper, 2012,Sandell and Peters, 2003). Other studies have suggested that selective axonal loss occurs in healthy aging, providing evidence of "resilience" of thick axons with thick myelin, combined with the loss of thin, poorly-myelinated axons (Marner, et al., 2003,Tang, et al., 1997). Ex vivo studies also pointed out that, despite alterations in the myelin sheaths, only a few nerve fibers showed axonal degeneration (Peters and Sethares, 2002). While FA is a sensitive marker for WM injury without specificity for axonal injury or demyelination, it has been suggested that increases in radial diffusivity and decreases in axial diffusivity correlate with demyelination processes and axonal damage, respectively (Song, et al., 2002,Wang, et al., 2014). Support for this hypothesis was also provided by histological analysis (Budde, et al., 2009,Hofling, et al., 2009,Kim, et al., 2006). Although DTI metrics are potentially sensitive measures of alterations in tissue microstructure, they are indirect measures of tissue integrity. Since DTI reflects the molecular diffusion of water, which is present in all tissue compartments, it is influenced by several microstructural factors that affect both the intraas well as the extracellular space, including myelin and axonal density, membrane integrity and axonal orientation dispersion (Beaulieu, 2002,Douaud, et al., 2011). As a consequence, the 
interpretation of DTI metrics based on the neurobiological mechanisms that determine their values is challenging. The interpretation of DTI data is further complicated by the local architecture of WM tracts. For example, in the case of crossing fibers, the major axis of the tensor model may not be parallel to the myelinated axons, the many separate tracts which propagate within the DTI voxel result in low local FA values (Pierpaoli, et al., 2001, Wheeler-Kingshott and Cercignani, 2009).

Diffusion-weighted ${ }^{1} \mathrm{H}$ magnetic resonance spectroscopy (DW-MRS) provides structural information exclusively related to the intracellular space by probing the diffusion properties of intracellular metabolites, such as total $\mathrm{N}$-acetylaspartate $(\mathrm{tNAA}=\mathrm{N}$-acetylaspartate $+\mathrm{N}$-acetyl aspartyl glutamate), total creatine $(\mathrm{tCr}=$ creatine + phosphocreatine $)$, and choline compounds (tCho) (Nicolay, et al., 2001,Posse, et al., 1993). The distribution of these metabolites across different cell types in the brain makes DW-MRS not only compartment-specific, but also specific or at least preferential for different cell types (Choi, et al., 2007,Le Belle, et al., 2002,Urenjak, et al., 1993). NAA and NAAG are known to be present almost exclusively inside neurons, and in white matter they are exclusively intra-axonal. tNAA diffusion properties in white matter are therefore solely dictated by the structural properties of intra-axonal components and by the properties of the intra-axonal medium, thus providing a specific marker to the axonal contribution to WM degeneration. Soluble Choline compounds are located mainly in glial cells (Choi, et al., 2007,Le Belle, et al., 2002,Urenjak, et al., 1993) and thus their diffusion properties report on structural changes in glia, resulting from, for example, inflammation (Ercan, et al., 2016). Creatine and phosphocreatine are present in both tissue types and are tightly linked to cell energetics through the creatine kinase cycle $(\mathrm{CK})$, and it has been shown that increase in energy demand modulates the diffusion coefficient of total creatine (Branzoli, et al., 2013). Previous observations have provided strong evidence for the important physiological role of the CK system for normal brain function, and suggested that energy dysfunction processes are crucially related to cell death not only in neurodegenerative disorders but even in normal aging (Andres, et al., 2008). So far, DW-MRS has been applied to study normal aging (Zheng, et al., 2012) as well as disease pathophysiology in brain 
tumors (Colvin, et al., 2008,Harada, et al., 2002), acute cerebral ischemia (Harada, et al., 2002,Zheng, et al., 2012), multiple sclerosis (Wood, et al., 2012), psychiatric disorders (Du, et al., 2013), and mitochondrial cytopathies (Liu, et al., 2011). Here we employed DW-MRS in combination with DTI in order to separately detect and quantify intra-axonal damage and demyelination in healthy aging in the anterior body of the corpus callosum (CC). The integrity of the $\mathrm{CC}$ plays a crucial role in cognitive and motor decline, since processes involved in crosshemispheric information exchange depend on the integrity of the callosal fibers (Banich, 1998,Reuter-Lorenz and Stanczak, 2000). Differences in the DTI metrics measured in the corpus callosum of healthy elderly have been reported in several DTI studies, yet the underlying biological mechanisms for these changes are still unknown (Billiet, et al., 2015,Fling, et al., 2011,Teipel, et al., 2010). In addition, the CC is a prominent white matter structure that is easy to identify on MRI images, and thus positioning of the volume of interest (VOI) for DW-MRS experiments in a consistent manner across subjects is relatively straightforward. The morphology of the CC allows a relatively easy estimate of the pure intra-axonal tNAA diffusivity, as well as accurate volumetric measurements, of interest as macroscopic correlates of the microstructural indices derived from the DW-MRS and DTI experiments.

In this study, we used DTI and DW-MRS to measure the diffusion properties of water, tNAA, tCr, and tCho in the CC of healthy elderly and young subjects at 7T. We combined the information from these two MR tools to discriminate between age-related myelin alterations and possible concomitant axonal or glial structural changes, and to obtain insight into possible cell energy alterations in normal aging. For an unbiased estimation of the intra-axonal diffusivity of tNAA, we analyzed the DW-MRS data using a model that provides the cytosolic diffusion coefficient of tNAA, free from confounds stemming from the curvature of the $\mathrm{CC}$ within the VOI and from microscopic fiber orientation dispersion (Ronen, et al., 2014). 


\section{Materials and methods}

\subsection{Human subjects}

Thirteen healthy young controls (mean age $27 \pm 3$ years, age range 23-32 years, 8 males, 5 females) and sixteen healthy elderly subjects (mean age $64 \pm 3$ years, age range 60-71 years, 10 males, 6 females) gave informed consent according to the procedures approved by the Leiden University Medical Center Institutional Review Board. Demographic characteristics are reported in Table 1. Subjects had no known history of neurological or psychiatric disorders.

\subsection{MRI scanner/Hardware}

Scans were performed on a 7 Tesla Achieva Philips whole-body MRI scanner (Philips Healthcare, Best, The Netherlands) equipped with gradient coils capable of a maximum gradient strength of 40 $\mathrm{mT} / \mathrm{m}$ and a slew rate of $200 \mathrm{~T} / \mathrm{m} / \mathrm{s}$. A head coil consisting of a quadrature birdcage transmit and 32-channel phased array receive were used for all measurements (Nova Medical Inc., Wilmington, MA, USA).

\section{$2.3 \quad$ MRI/MRS protocol}

Anatomical images: A short survey scan and a sensitivity encoding (SENSE) reference scan were followed by a $3 \mathrm{D} \mathrm{T}_{1}$-weighted gradient echo acquisition to allow for accurate planning of the spectroscopy volume of interest (VOI). Imaging parameters were: field-of-view (AP, FH, LR): $246.4 \times 246.4 \times 174.0 \mathrm{~mm}^{3}$, resolution $1 \times 1 \times 1 \mathrm{~mm}^{3}, \mathrm{TR} / \mathrm{TE}=4.7 \mathrm{~ms} / 2.1 \mathrm{~ms}$.

DTI protocol: Multi-slice single-shot 2D spin-echo echo planar imaging (EPI) was performed with the following data acquisition parameters: 60 slices of $2 \mathrm{~mm}$ thickness, TR/TE $=9100 \mathrm{~ms} / 58 \mathrm{~ms}$, field of view of $224 \times 224 \times 120 \mathrm{~mm}^{3}$ with an in-plane data matrix of $112 \times 112$, resulting in a data set with resolution of $2 \times 2 \times 2 \mathrm{~mm}^{3}, 15$ diffusion weighting directions with $\mathrm{b}=1000 \mathrm{~s} / \mathrm{mm}^{2}$. Fat suppression was performed using spectral inversion recovery (SPIR). Parallel imaging was 
performed using SENSE with a reduction factor of 3 along the phase encoding direction (AP).

DW-MRS protocol: A single voxel, point resolved spectroscopy (PRESS) sequence with a bipolar diffusion weighting gradient scheme was used. A VOI of 30 (AP) x 15 (LR) x8 (FH) $\mathrm{mm}^{3}$ was positioned in the anterior part of the body of the CC (Insets of Figure 1). Sequence parameters were: $\mathrm{TR}=3$ cardiac cycles (cardiac triggering was performed using the peripheral pulse unit), TE $=121$ ms, spectral width $\mathrm{SW}=3 \mathrm{kHz}$, and number of acquired complex sample points $\mathrm{NP}=2048$.

Diffusion weighting was applied in an interleaved way in two directions with respect to the VOI: left-right ( $\mathbf{g}_{(0,0,1)}$, mostly parallel to the callosal fibers), and one that formed a $45^{\circ}$ angle between the superior-inferior and anterior-posterior directions of the VOI $\left(\mathbf{g}_{(1,1,0)}\right.$, mostly perpendicular to the callosal fibers). The VOI position and the gradient directions are shown in Figure 1 (insets). The diffusion weighting was applied using a diffusion time $\Delta=60.5 \mathrm{~ms}$, duration of bipolar diffusion group: $\delta=34 \mathrm{~ms}$, bipolar gap: $18 \mu \mathrm{s}$ and 4 increasing gradient strengths $\mathrm{g}$ corresponding to $\mathrm{b}$ values $330,960,1900,3200 \mathrm{~s} / \mathrm{mm}^{2}$, and $660,1920,3800,6400 \mathrm{~s} / \mathrm{mm}^{2}$ for the $(0,0,1)$ and $(1,1,0)$ directions, respectively. 32 spectra were acquired for each direction and b value. Water suppression was applied using a frequency selective excitation pulse followed by a dephasing gradient before metabolite excitation. The water signal was only partially suppressed to allow for a sufficient residual water signal for individual phasing of each spectrum. Non-water suppressed spectra were acquired under similar conditions for eddy current corrections. The total duration of the DW-MRS protocol was approximately 15 minutes.

\subsection{Post-processing and analysis}

Image processing: $\mathrm{T}_{1}$-weighted images were used for tissue segmentation within the VOI, and further processed in FreeSurfer (http://surfer.nmr.mgh.harvard.edu/) to calculate the intracranial volume, total brain volume, and CC volumes for each subject. To evaluate the callosal atrophy due to aging, the CC volumes were normalized according to the intracranial volume of the same subject.

The maps of the main eigenvector $\left(\mathbf{e}_{1}\right)$ were derived and confined to the VOI using MIPAV 
(Medical Image Processing, Analysis and Visualization) software, JIST (Java Image Science Toolbox) and in-house developed MATLAB® codes (Mathworks Inc., Natick MA, USA) as described in (Wood, et al., 2015). These maps were used in the modeling procedure described below.

Average DTI volumes were motion corrected with ExploreDTI, and DTI metrics were derived using the DTI toolbox of the FMRIB Software Library (FSL release 5.0, http://www.fmrib.ox.ac.uk/fsl/). The DTI metrics were further analyzed with tract based spatial statistics (TBSS) (Smith, et al., 2006). Statistical differences between older and younger subjects were assessed in FA-MNI152 standard space using 5000 permutations and were corrected for multiple comparisons based on threshold-free cluster enhancement (Winkler, et al.). Three young and one old volunteer were excluded from TBSS analysis due to the poor registration to FAMNI152. $\mathrm{T}_{1}$-volumes were registered to MNI152 space first by affine transformation using FSL FLIRT (Jenkinson, et al., 2002,Jenkinson and Smith, 2001), and then by a non-rigid registration using FNIRT(Andersson, et al., 2010). The same transformation matrices were then used to register the DW-MRS VOI to the MNI152 standard space.

$D W$-MRS processing: The diffusion-weighted spectra were quantified using LCModel (Provencher, 2001) using an appropriate basis set. Diffusion coefficients for tNAA in the two gradient directions, $\mathrm{D}_{(0,0,1)}(\mathrm{tNAA})$ and $\mathrm{D}_{(1,1,0)}(\mathrm{tNAA})$, were estimated by fitting the tNAA peak estimates from LCModel to monoexponential decay functions as shown for one subject in Figure 2. $\mathrm{D}_{(0,0,1)}(\mathrm{tCr})$, $\mathrm{D}_{(1,1,0)}(\mathrm{tCr})$, and $\mathrm{D}_{(0,0,1)}(\mathrm{tCho}), \mathrm{D}_{(1,1,0)}(\mathrm{tCho})$ were estimated similarly. The cytosolic diffusion coefficient of tNAA, D(tNAA), was estimated by fitting the tNAA LCModel data to a model described in detail in (Ronen, et al., 2014). Briefly, the model assumes the tNAA to be exclusively intra-axonal, and the axons to be curved cylinders with a known diameter distribution (Aboitiz, et al., 1992). The effect of the macroscopic curvature of the CC within the VOI on the diffusionweighted data in both diffusion weighted directions is accounted for by using the distribution of angles between the main eigenvectors $\mathbf{e}_{1}$ of the DTI data and the diffusion weighting directions. The 
experimental data is fitted to the model using two fitting variables: D(tNAA), which is independent of the curvature of the tract within the VOI, and the microscopic axonal angular dispersion $\varphi$, which describes the average fanning of individual axons about the main propagation direction within a DTI voxel. Student t-test were performed in order to evaluate possible statistical differences between the two groups. The significance level was set to $\mathrm{p}=0.05$.

\section{Results}

Table 1 summarizes the demographic characteristics of young and healthy elderly subjects, as well as the average voxel tissue composition of the two groups, and the CC normalized volumes. Small, not statistically significant differences, were observed between the two groups in the WM fraction (young subjects: WM fraction 64\%, elderly subjects: WM fraction 68\%) and CSF fractions (young subjects: WM fraction 29\%, elderly subjects: WM fraction $\sim 24 \%$ ), while differences in GM fraction were negligible (GM fraction 8\% in both groups). However, when the WM and GM fractions were normalized over the brain tissue percentage $(100-\operatorname{CSF}(\%))$, no differences could be observed in the tissue composition (WM $\sim 90 \%, \mathrm{GM} \sim 10 \%$ in both groups). The difference in normalized callosal volume between the two groups was not statistically significant $(\mathrm{p}=0.11)$.

Figure 3a shows an FA map of a coronal section of the corpus callosum calculated for one of the younger subjects, overlaid with the representation of the main eigenvectors of the diffusion tensor for each voxel, and with the DWS VOI. Figure $3 \mathrm{~b}$ and $\mathrm{c}$ show histograms of the distributions of the angles $\theta_{1}$ and $\theta_{2}$ of the main eigenvector $\mathbf{e}_{1}$ and the gradients $\mathbf{g}_{(0,0,1)}$ and $\mathbf{g}_{(1,1,0)}$, respectively, calculated within the masked VOI for the same subject. Histogram bins are of $1^{\circ}$. The two distributions show the highest probability around $\pm 13^{\circ}$ and around $95^{\circ}$, for $\theta_{1}$ and $\theta_{2}$ respectively, consistently with the CC morphology.

Figure 4 show maps of statistically significant differences in DTI measures overlaid on the MNI152 
$\mathrm{T}_{1}$-weighted image, and the cumulative DW-MRS VOI (i.e. the sum of all the VOIs for each patient, following the transformation to MNI152 coordinates). Significantly lower FA, higher MD and higher radial diffusivity (RD) in the older subjects group compared to the younger subjects group were found throughout white matter, including within the DW-MRS VOI. In contrast, no significant differences in AD were measured in the VOI.

Examples of diffusion-weighted spectra acquired in one subject with diffusion gradients applied in the directions parallel and perpendicular to the $\mathrm{CC}$ fibres at low and high $\mathrm{b}$ values are shown in Figure 1. The signal decay induced by the diffusion weighting in the direction parallel to the callosal fibres was much steeper than when the gradient was applied in the direction perpendicular to the fibres. This is due to the fact that the molecular motion along the axonal direction is essentially unrestricted, as opposed to the motion perpendicular to the axonal membrane. The linewidth of tNAA was $~ 12 \mathrm{~Hz}$, and the Cramer-Rao lower bound (CRLB) was $<15 \%$ for all diffusion weighting conditions for tNAA and $\mathrm{tCr}$, and $<20 \%$ for tCho. Figure 2 shows the tNAA normalized signal decay as derived from LCModel analysis, plotted on a logarithmic scale, as a function of the $\mathrm{b}$ value, for the two directions $(0,0,1)$ and $(1,1,0)$ (closed and open symbols, respectively). Both the modelled free tNAA diffusion coefficient, D(tNAA), and the tNAA diffusion coefficient measured in the direction of the callosal fibers, $\mathrm{D}_{(0,0,1)}(\mathrm{tNAA})$, were found to be slightly smaller in healthy elderly subjects compared to young controls, but these differences did not reach statistical significance. Neither the tNAA diffusion coefficient measured in the direction perpendicular to the fibers, $\mathrm{D}_{(1,1,0)}(\mathrm{tNAA})$, nor the the axonal microscopic angular dispersion angle $\varphi$ differed between the two groups.

The $\mathrm{tCr}$ axial diffusion coefficient $\mathrm{D}_{(0,0,1)}(\mathrm{tCr})$ was found to be smaller in healthy elderly subjects ( $\mathrm{p}$ $=0.058$, while the other $\mathrm{tCr}$ diffusion parameters did not change between the two groups.

None of the tCho diffusion properties was found to differ significantly between the two groups.

Table 2 summarizes the results for metabolite diffusion parameters in young and healthy elderly 
subjects.

\section{Discussion}

In this study we used a combination of tools sensitive to the microstructural features of neural tissue to investigate age-related changes in normal-appearing white matter in healthy aging. The novel feature we introduced here is the incorporation of DW-MRS, a compartment and cell-type measure for specific alterations in the intracellular space of axons and glia. The observed differences in water MD (higher in older adults) and in water FA (lower in older adults) in the CC are consistent with previous studies suggesting the onset of demyelination processes in the seventh decade of age or later in healthy elderly (Salat, et al., 2005,Sullivan and Pfefferbaum, 2006). The estimated water RD values were found to be greater in elderly subjects, while no significant differences in the water AD were observed in the DWS voxel, also in accordance with literature reports (Burzynska, et al., 2010,Coutu, et al., 2014,Madden, et al., 2012). The observed differences in water diffusion properties of the elderly cannot be solely related to a loss of axonal fibers leading to an increase in the extracellular space, since the older adults did not show evidence of severe atrophy of the CC, as expected in subjects in this age range (60-70 years), and the differences in callosal volumes were below the threshold of statistical significance. In some previous studies the DTI results were interpreted as a consequence of the fact that in healthy aging demyelination processes are dominant with respect to other possible microstructural WM damages such as disruption of intra-axonal components, based on the hypothesis that water $\mathrm{AD}$ changes should reflect ongoing intra-axonal damage (Madden, et al., 2012). However, the assumption that the water AD can be unequivocally associated with degeneration processes occurring in the intra-cellular space as suggested previously (Hofling et al., 2009) seems to be rather simplistic if the complexity of fiber organization is taken 
into account. Even in a region of the WM such as the $\mathrm{CC}$ where areas with no macroscopic intersection of fiber bundles can be isolated, the presence of microscopic fiber bending and fiber crossing makes the interpretation of the $\mathrm{AD}$ parameter more complicated, since it may also be susceptible to demyelination effects (Ronen et al., 2013a; Wheeler-Kingshott and Cercignani, 2009).

The hypothesis that water $\mathrm{AD}$ alone cannot be interpreted as reflecting purely intra-axonal changes was recently suggested in a study by Wood et al., where an increase in water AD and a concomitant decrease in tNAA axial diffusivity were observed in the CC of multiple sclerosis (MS) patients using a methodology similar to the one presented here. In Wood et al., the diffusion tensor of tNAA was calculated to yield, among the other metrics, the axial diffusivity of tNAA. Although this measure could be partially affected by the macroscopic curvature of the $\mathrm{CC}$ in the spectroscopic VOI, as well as by the microscopic axonal misalignment in the DTI voxel, an opposite trend was observed in water and tNAA axial diffusivity, showing that these two metrics are not markers of the same biological processes. This point is further emphasized by the confirmation here of the existence of significant axonal misalignment $(\varphi \sim 12 \mathrm{deg})$, already shown by DW-MRS and histology (Ronen et al., 2013a), and known to affect DTI measurements as well (Sotiropoulos, et al., 2016).

In the current study, we estimated the cytosolic tNAA diffusion coefficient within axons, which is not affected by the interactions with the cellular walls, or by fiber geometry, and it is thus a pure measure of intra-axonal integrity. Our results are the first to show a decrease in $\mathrm{D}_{(0,0,1)}(\mathrm{tNAA})$, as well as a decrease in $\mathrm{D}(\mathrm{tNAA})$, in an elderly population; however, the results did not reach statistical significance. These differences, if confirmed, may be related to the presence of structural disruptions associated with axonal degeneration, or pruning of thinner axons, and the very small effect is in line with the fact that our group included healthy aging subjects in the seventh decade. The population size in this study was similar to that in a previous DW-MRS study in MS that investigated axonal differences in normal appearing white matter in the CC (Wood, et al., 2012). In 
both studies, significant effects on DTI measures such as FA and RD were detected. In our current study, an optimized acquisition scheme was employed that corrects for inter-subject variability in the CC curvature using a previously demonstrated model. Yet, the differences in D(tNAA) in the $\mathrm{CC}$ between the two age populations were negligible. Our results suggest that the intra-axonal damage in the $\mathrm{CC}$ of healthy subjects in this age range is small $(<10 \%)$, in line with the results derived by Peters and Sethares in 2002 from histological measurements in normally-aging macaques, and supporting the idea that cognitive impairments are more strongly associated with axonal integrity rather than with demyelination processes(Sandell and Peters, 2003).

The creatine axial diffusivity $\mathrm{D}_{(0,0,1)}(\mathrm{tCr})$ was found to be lower in healthy elderly subjects compared to younger controls but this finding did not reach statistical significance $(\mathrm{p}=0.058)$. This finding may be linked to the processes involving adenosine triphosphate (ATP) production and utilization in the brain, which are closely connected to the conversion of $\mathrm{PCr}$ to $\mathrm{Cr}$ through the $\mathrm{CK}$ cycle (Wallimann, et al., 1992). By enabling saltatory conduction, myelination is also thought to reduce ATP consumption and, in turn, to be related to axonal energy balance (Saab, et al., 2013). A lower ATP production rate in aging brains was further corroborated by a study using phosphorus MRS at 7T (Zhu, et al., 2015). Recent studies have convincingly shown that glial cells, especially oligodendrocytes at the axonal level and astrocytes at the synaptic level, supply lactate to neurons as a metabolic substrate for mitochondria to generate ATP, and that this process is altered by demyelination (Saab, et al., 2013). The demyelination processes affecting the white matter of healthy elderly subjects may, therefore, be indirectly reflected in abnormalities in the diffusion of $\mathrm{tCr}$ as a consequence of an impaired energy metabolism, possibly yielding an increase in the $\mathrm{PCr} / \mathrm{Cr}$ ratio, or a dysfunction in the trafficking of these metabolites between glia and neurons (Andres, et al., 2008).

Taken together, our results on tNAA and tCr diffusion support the hypothesis that the two major oligodendrocyte functions of myelination and axonal support are distinct, as suggested by a previous study where no perturbation of axonal integrity was observed in a model of truly 
dysmyelinated mutant mouse (Griffiths, et al., 1998).

The high specificity of MRS techniques is provided at the cost of an intrinsic low signal-to-noise ratio (SNR), which is further exacerbated by the addition of diffusion weighting and error propagation in the calculation of diffusion measures. The reproducibility of the model employed here for calculating metabolite diffusion metrics was tested previously in a study investigating the inter- and intra-subject variability of both empirical and modelled diffusion properties of tNAA, and evaluating the optimized protocol to be used in clinically relevant acquisition times in the same region of interest (Wood, et al., 2015). The acquisition scheme employed in this study was carefully evaluated following the guidelines reported in Wood et al. 2015. Power calculations in the same study showed that, based on the suggested acquisition scheme, at $7 \mathrm{~T}$ the minimum number of subjects in each group necessary to detect a $10 \%$ difference in D(tNAA), ADC(tNAA) and $\mathrm{ADC}(\mathrm{tCr})$ between two groups is 13,12 , and 7 , respectively. The acquisition scheme employed in the current study is expected to give less variability than the one in (Wood, et al., 2015), as it employs similar number of averages with one more b-value. This emphasizes the accuracy and reproducibility of DW-MRS measurements at 7T.

One of the limitations of this study is that, due to the non-negligible chemical shift displacement related to the relatively long refocusing RF pulses in the PRESS sequence, the VOI localization was optimized for tNAA, but not for $\mathrm{tCr}$. Therefore, $\mathrm{D}_{(0,0,1)}(\mathrm{tCr})$ may be contaminated by $\mathrm{D}_{(1,1,0)}(\mathrm{tCr})$ to a larger extent than in the case of tNAA, due to the imperfect alignment of the VOI with the fibers. Another limitation is the impossibility of excluding completely grey matter contamination from the VOI, due to the shape of the corpus callosum and the minimum size of the VOI required to achieve a reasonable spectral signal-to-noise ratio. Although an average fraction of GM of about $8 \%$ was present in the VOI of both young and elderly, it is very likely that this may introduce a small bias in the estimation of the metabolite diffusion properties, albeit without affecting the comparison between groups. Additional factors that may have impacted on the statistical significance of our results, and that should be improved in future studies, are the relatively small number of subjects 
who were recruited, the sub-optimal resolution for the DTI acquisition employed due to time constraints, and the localized spectroscopy method used. Improvements to the latter could be implemented using, for example, a diffusion-sensitized semi-localized by adiabatic selective refocusing (SEMI-LASER) technique that would result in a reduced chemical shift displacement (Scheenen et al.) and $\mathrm{B}_{1}$ field inhomogeneity compared to the PRESS technique. Although 7T allows higher SNR and higher resolution, due to the limited time allowed for this study the DTI resolution was sacrificed in order to allow enough time for an optimized DW-MRS acquisition and the other scans included in the protocol.

\section{Conclusion}

In this work we explored age-related microstructural changes in the corpus callosum in vivo with an approach that combines diffusion tensor imaging (DTI) with diffusion-weighted MRS (DW-MRS) at ultrahigh field. The combination of the overall sensitivity to microstructural changes provided by DTI, and the specificity of DW-MRS to the intracellular space, provides a unique possibility for invivo characterization of subtle compartment-specific changes in tissue microstructure in normal aging. Furthermore, the specificity or preferentiality of certain metabolites to either neurons/axons or to glia endows this approach with an additional level of characterization, unmet by other MRbased methods. Our results show that the intra-axonal microstructural changes as measured by tNAA diffusivity, if present, are below the level of detectability with our DW-MRS protocol in healthy elderly in the age range investigated, suggesting that the intra-axonal integrity is mainly preserved in this population. This result requires confirmation in further studies employing larger population groups for establishing subtle differences in these intracellular measures. Abnormal changes in the $\mathrm{tCr}$ diffusivity may be interpreted as a reduction in the energy metabolism related to an ongoing CK dysfunction and coupled to myelin synthesis and transport. Further experiments are nevertheless needed to corroborate this hypothesis. Our findings will be substantiated with 
preclinical studies of animal models of disease combined with histology for comparison of DWMRS metrics with a gold standard.

Table 1: Demographic characteristics, spectroscopic voxel tissue composition and normalized callosal volumes of healthy elderly subjects and healthy young controls. Values are the mean \pm standard deviation.

\begin{tabular}{lccc}
\hline & Young & Elderly & p-value \\
\hline Age (years) & $27 \pm 3$ & $64 \pm 3$ & 1 \\
Gender (male/female) & $8 / 5$ & $10 / 6$ & 0.96 \\
Voxel WM composition (\%) & $63 \pm 8$ & $68 \pm 7$ & 0.11 \\
Voxel GM composition (\%) & $8 \pm 3$ & $8 \pm 3$ & 0.95 \\
Voxel CSF composition (\%) & $28 \pm 8$ & $24 \pm 7$ & 0.10 \\
CC volume (\%) & $0.24 \pm 0.02$ & $0.22 \pm 0.03$ & 0.11 \\
\hline
\end{tabular}


Table 2: tNAA, tCr, and tCho group average diffusion properties and standard deviation of the microscopic axonal orientation distribution measured in the spectroscopic voxel. Values are the mean \pm standard deviation.

\begin{tabular}{lccc}
\hline & Young & Elderly & p value \\
\hline $\mathrm{D}(\mathrm{tNAA})\left(\mu \mathrm{m}^{2} / \mathrm{ms}\right)$ & $0.53 \pm 0.04$ & $0.51 \pm 0.04$ & 0.21 \\
$\mathrm{D}_{(0,0,1)}(\mathrm{tNAA})\left(\mu \mathrm{m}^{2} / \mathrm{ms}\right)$ & $0.39 \pm 0.04$ & $0.37 \pm 0.04$ & 0.12 \\
$\mathrm{D}_{(1,1,0)}(\mathrm{tNAA})\left(\mu \mathrm{m}^{2} / \mathrm{ms}\right)$ & $0.08 \pm 0.02$ & $0.08 \pm 0.02$ & 0.79 \\
$\mathrm{D}_{(0,0,1)} / \mathrm{D}_{(1,1,0)}(\mathrm{tNAA})$ & $4.9 \pm 1.1$ & $4.8 \pm 1.4$ & 0.78 \\
$\mathrm{ADC}(\mathrm{tNAA})$ & $0.24 \pm 0.02$ & $0.23 \pm 0.02$ & 0.18 \\
$\sigma_{\varphi}(\mathrm{deg})$ & $12 \pm 5$ & $12 \pm 5$ & 0.77 \\
$\mathrm{D}_{(0,0,1)}(\mathrm{tCr})\left(\mu \mathrm{m}^{2} / \mathrm{ms}\right)$ & $0.27 \pm 0.04$ & $0.24 \pm 0.04$ & 0.058 \\
$\mathrm{D}_{(1,1,0)}(\mathrm{tCr})\left(\mu \mathrm{m}^{2} / \mathrm{ms}\right)$ & $0.11 \pm 0.03$ & $0.11 \pm 0.03$ & 0.96 \\
$\mathrm{D}_{(0,0,1)} / \mathrm{D}_{(1,1,0)}(\mathrm{tCr})$ & $2.5 \pm 0.6$ & $2.2 \pm 0.8$ & 0.30
\end{tabular}


$\mathrm{ADC}(\mathrm{tCr})$

$\mathrm{D}_{(0,0,1)}(\mathrm{tCho})\left(\mu \mathrm{m}^{2} / \mathrm{ms}\right)$

$0.19 \pm 0.03$

$0.18 \pm 0.05$

$0.17 \pm 0.04$

0.56

$\mathrm{D}_{(1,1,0)}(\mathrm{tCho})\left(\mu \mathrm{m}^{2} / \mathrm{ms}\right)$

$0.11 \pm 0.02$

$0.10 \pm 0.03$

0.90

$\mathrm{D}_{(0,0,1)} / \mathrm{D}_{(1,1,0)}(\mathrm{tCho})$

$1.8 \pm 0.7$

$1.7 \pm 0.8$

0.60

ADC(tCho)

$0.14 \pm 0.03$

$0.14 \pm 0.03$

0.56

Figures 

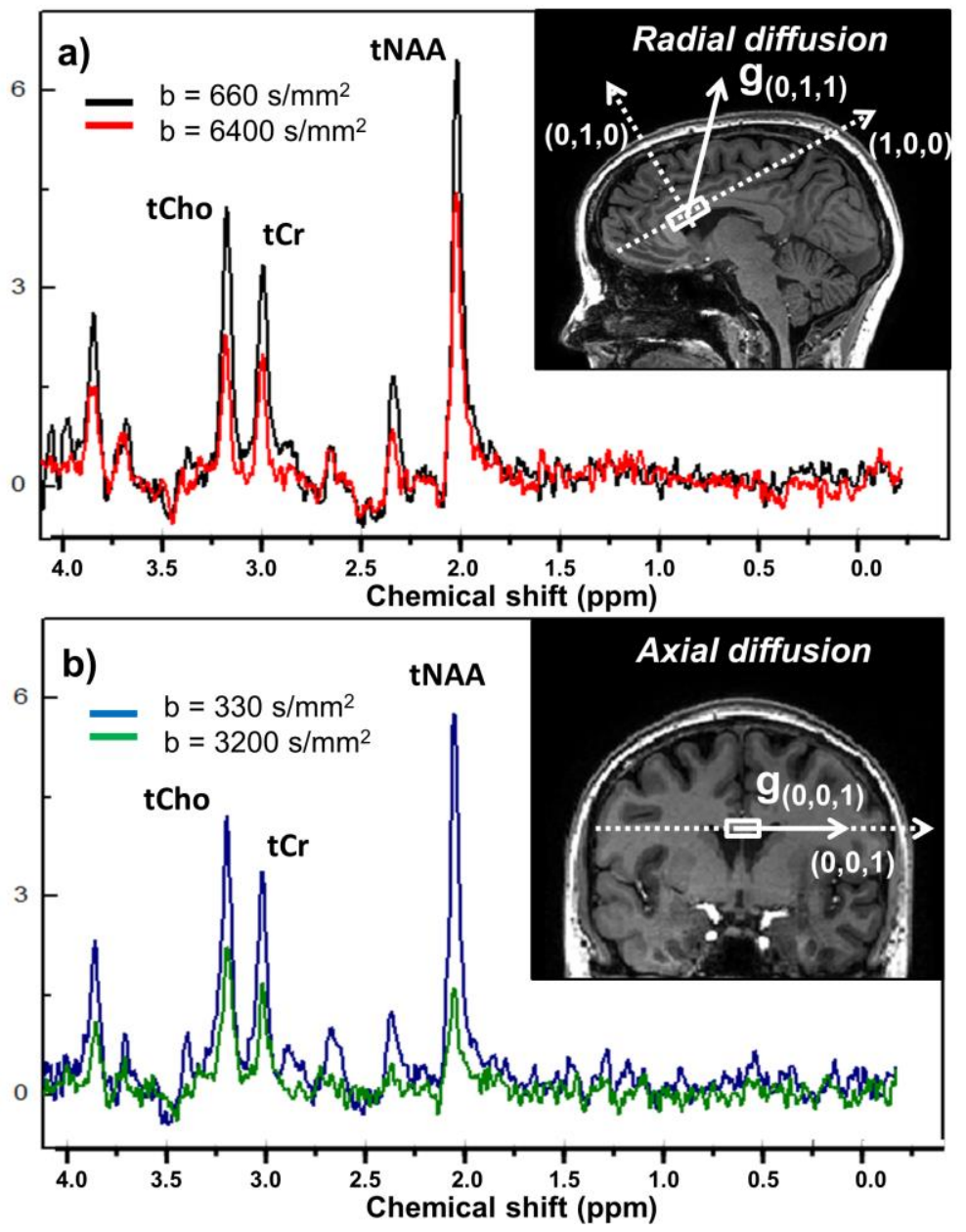

Figure 1: DW-MRS spectra and volume of interest. Examples of spectra acquired from a single subject with diffusion weighting applied perpendicular $\left(\mathbf{a}, \mathrm{b}=660 \mathrm{~s} / \mathrm{mm}^{2}\right.$ and $\left.\mathrm{b}=6400 \mathrm{~s} / \mathrm{mm}^{2}\right)$ and parallel $\left(\mathbf{b}, \mathrm{b}=300 \mathrm{~s} / \mathrm{mm}^{2}\right.$ and $\left.\mathrm{b}=1900 \mathrm{~s} / \mathrm{mm}^{2}\right)$ to the callosal fibers. A line broadening of $4 \mathrm{~Hz}$ was applied. Insets: the volume of interest (VOI), as seen from a sagittal (a) and a coronal (b) view of a T1-weighted image, and the two diffusion weighting directions $\mathbf{g}_{[0,1,1]}$ and $\mathbf{g}_{[0,0,1]}$ in the VOI coordinate system. 


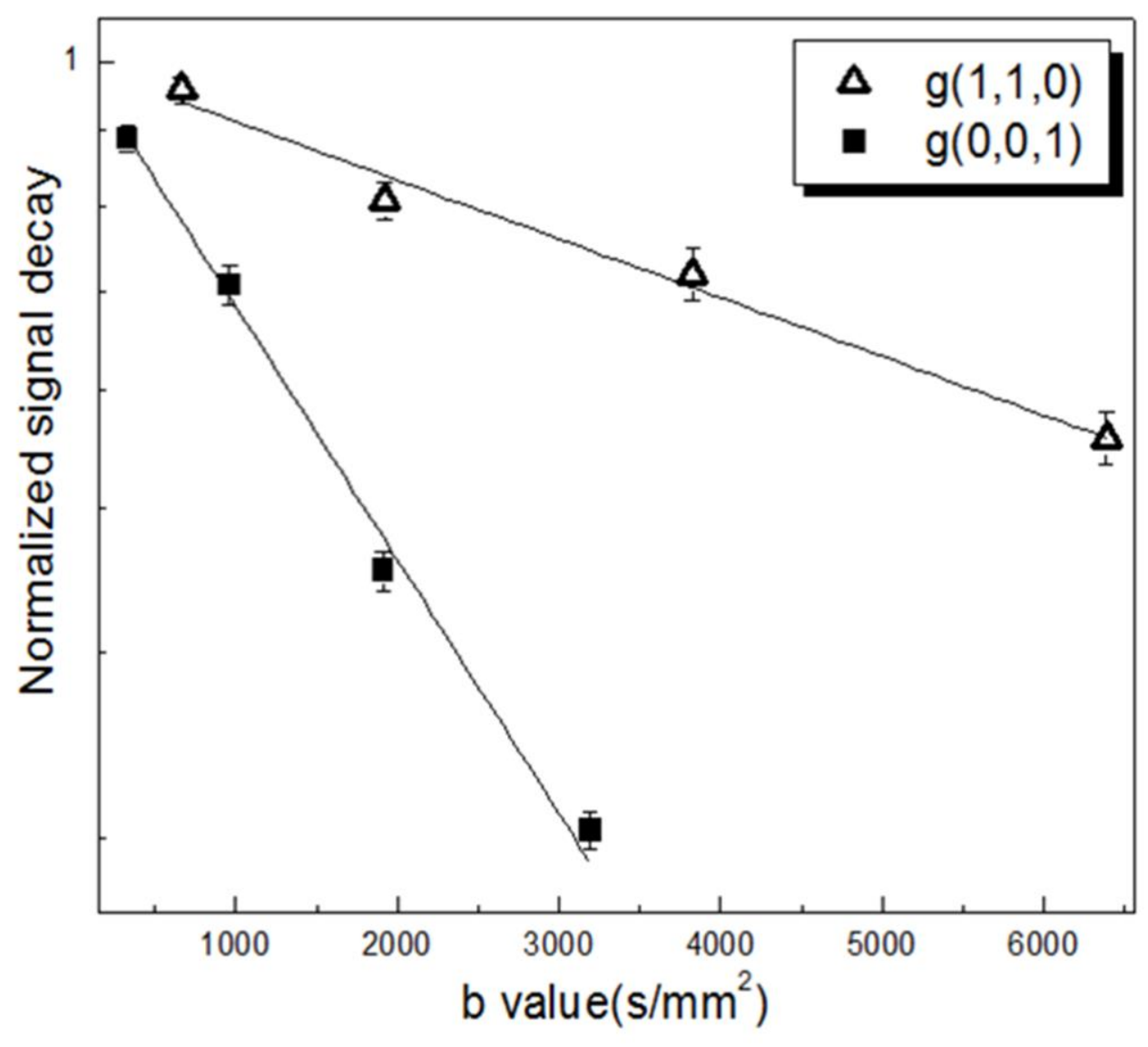

Figure 2: tNAA signal decay induced by diffusion weighting. tNAA normalized signal decay, measured for one subject, plotted in a logarithmic scale as a function of the b value and for the two directions $(0,0,1)$ (filled squares) and $(1,1,0)$ (open triangles). The signal decay induced by the diffusion weighting in the direction parallel to the callosal fibers $(0,0,1)$ was much steeper than when the gradient was applied in the direction perpendicular to the fibers $(1,1,0)$. This is due to the fact that the molecular motion along the axonal direction is essentially unrestricted, as opposed to the motion perpendicular to the axonal membrane. Solid lines represent fits to single exponentials. Error bars represent the Cramer-Rao Lower Bounds (CRLB) derived with LCModel. 
a
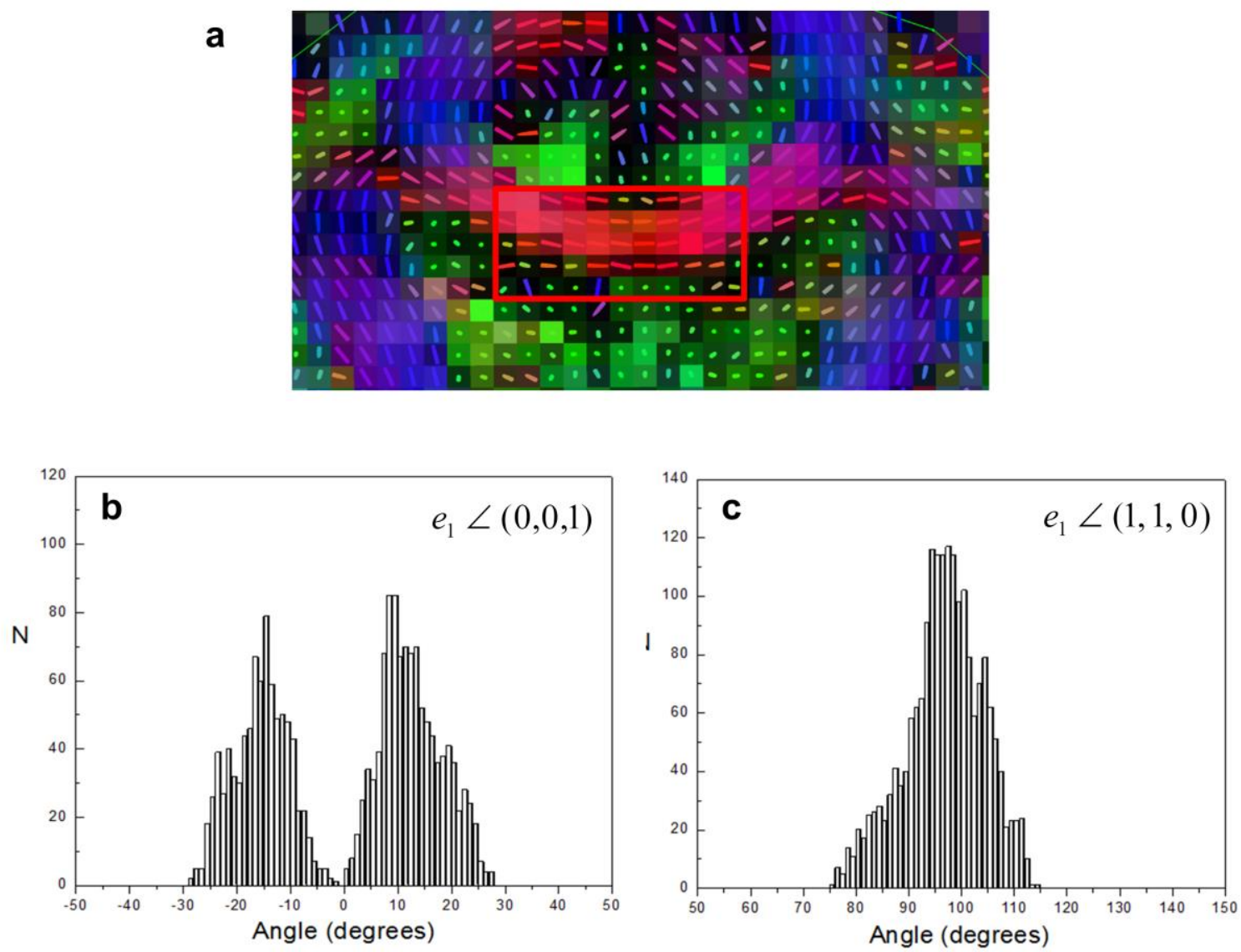

Figure 3: Macroscopic fiber orientation in the corpus callosum. a) Coloured FA map of a coronal section of the corpus callosum of one of the younger subjects overlaid with the representation of the main eigenvectors $\mathbf{e}_{1}$ of the diffusion tensor for each voxel. The red box represents the outline of the DWS VOI. b) Distribution of the angle $\theta_{1}$ between the main eigenvector $\mathbf{e}_{1}$ and $\mathbf{g}_{(0,0,1)}$. c) Distribution of the angle $\theta_{2}$ between the main eigenvector $\mathbf{e}_{1}$ and $\mathbf{g}_{(1,1,0)}$. 
FA
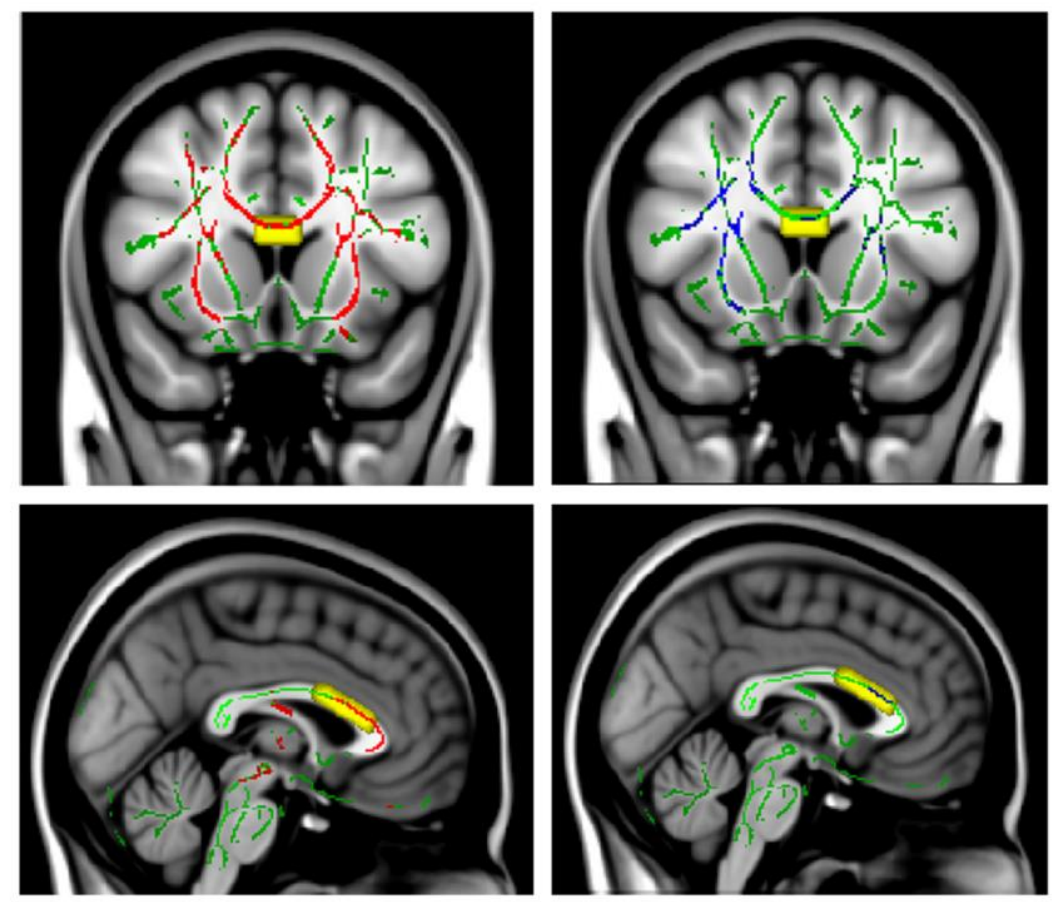

MD

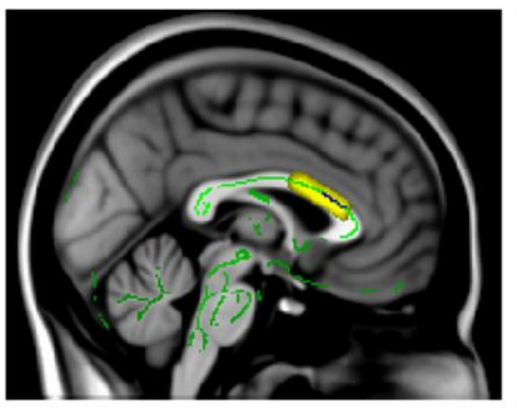

RD
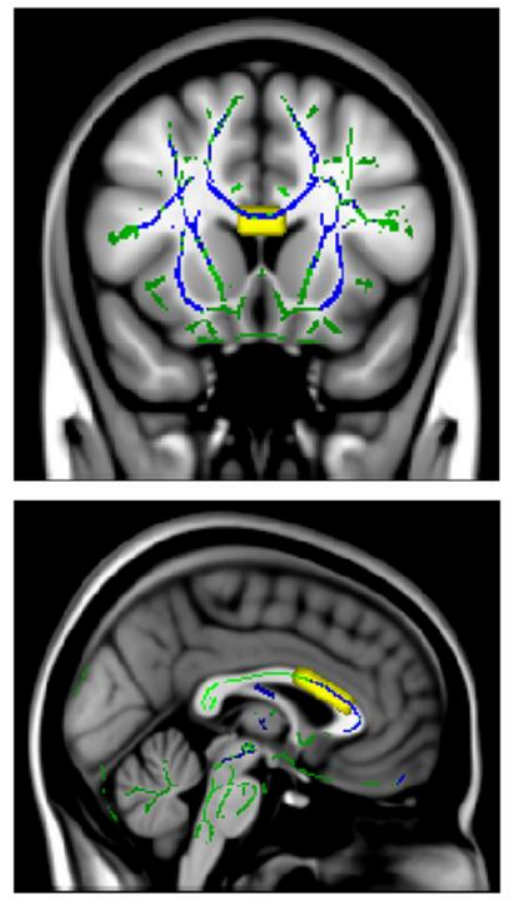

Figure 4: Differences in DTI metrics in healthy elderly versus younger controls. TBSS results showing regions with statistically significant differences in DTI measures in the white matter skeleton of older adults and younger controls ( $\mathrm{p}<0.05)$. Maps are shown on one sagittal (top row) and one coronal (bottom row) slice in MNI152 space. The mean FA skeleton is shown in green, regions with higher values in the older subjects compared to younger controls are shown in blue and regions with lower values in older subjects compared to younger controls are shown in red. Cumulative VOIs chosen for DW-MRS are shown in yellow. 
We thank the support of the IHU and the following grants: Institut des neurosciences translationnelle - ANR-10-IAIHU-06, and Infrastructure d'avenir en Biologie Santé - ANR-11INBS-0006.

\section{References}

Aboitiz, F., Scheibel, A.B., Fisher, R.S., Zaidel, E. 1992. Fiber composition of the human corpus callosum. Brain Res 598(1-2), 143-53.

Andersson, J., Jenkinson, M., Smith, S. 2010. Non-linear registration, aka spatial normalisation. FMRIB technical report TR07JA2.

Andres, R.H., Ducray, A.D., Schlattner, U., Wallimann, T., Widmer, H.R. 2008. Functions and effects of creatine in the central nervous system. Brain Res Bull 76(4), 329-43.

Banich, M.T. 1998. The missing link: the role of interhemispheric interaction in attentional processing. Brain and cognition 36(2), 128-57. doi:10.1006/brcg.1997.0950.

Beaulieu, C. 2002. The basis of anisotropic water diffusion in the nervous system - a technical review. NMR Biomed 15(7-8), 435-55.

Billiet, T., Vandenbulcke, M., Madler, B., Peeters, R., Dhollander, T., Zhang, H., Deprez, S., Van den Bergh, B.R., Sunaert, S., Emsell, L. 2015. Age-related microstructural differences quantified using myelin water imaging and advanced diffusion MRI. Neurobiol Aging 36(6), 2107-21. doi:10.1016/j.neurobiolaging.2015.02.029.

Branzoli, F., Techawiboonwong, A., Kan, H., Webb, A., Ronen, I. 2013. Functional diffusionweighted magnetic resonance spectroscopy of the human primary visual cortex at $7 \mathrm{~T}$. Magn Reson Med 69(2), 303-9. doi:10.1002/mrm.24542.

Budde, M.D., Xie, M., Cross, A.H., Song, S.K. 2009. Axial diffusivity is the primary correlate of axonal injury in the experimental autoimmune encephalomyelitis spinal cord: a quantitative pixelwise analysis. The Journal of neuroscience : the official journal of the Society for Neuroscience 29(9), 2805-13. doi:10.1523/jneurosci.4605-08.2009.

Burzynska, A.Z., Preuschhof, C., Backman, L., Nyberg, L., Li, S.C., Lindenberger, U., Heekeren, H.R. 2010. Age-related differences in white matter microstructure: region-specific patterns of diffusivity. Neuroimage 49(3), 2104-12. doi:10.1016/j.neuroimage.2009.09.041.

Choi, J.K., Dedeoglu, A., Jenkins, B.G. 2007. Application of MRS to mouse models of neurodegenerative illness. NMR Biomed 20(3), 216-37. doi:10.1002/nbm.1145.

Colvin, D.C., Yankeelov, T.E., Does, M.D., Yue, Z., Quarles, C., Gore, J.C. 2008. New insights into tumor microstructure using temporal diffusion spectroscopy. Cancer Res 68(14), 59417.

Coutu, J.P., Chen, J.J., Rosas, H.D., Salat, D.H. 2014. Non-Gaussian water diffusion in aging white matter. Neurobiol Aging 35(6), 1412-21.

Douaud, G., Jbabdi, S., Behrens, T.E., Menke, R.A., Gass, A., Monsch, A.U., Rao, A., Whitcher, B., Kindlmann, G., Matthews, P.M., Smith, S. 2011. DTI measures in crossing-fibre areas: increased diffusion anisotropy reveals early white matter alteration in MCI and mild Alzheimer's disease. Neuroimage 55(3), 880-90. doi:10.1016/j.neuroimage.2010.12.008.

Du, F., Cooper, A.J., Thida, T., Shinn, A.K., Cohen, B.M., Ongur, D. 2013. Myelin and axon 
abnormalities in schizophrenia measured with magnetic resonance imaging techniques. Biol Psychiatry 74(6), 451-7.

Ercan, E., Magro-Checa, C., Valabregue, R., Branzoli, F., Wood, E.T., Steup-Beekman, G.M., Webb, A.G., Huizinga, T.W., van Buchem, M.A., Ronen, I. 2016. Glial and axonal changes in systemic lupus erythematosus measured with diffusion of intracellular metabolites. Brain : a journal of neurology. doi:10.1093/brain/aww031.

Fling, B.W., Walsh, C.M., Bangert, A.S., Reuter-Lorenz, P.A., Welsh, R.C., Seidler, R.D. 2011. Differential callosal contributions to bimanual control in young and older adults. Journal of cognitive neuroscience 23(9), 2171-85. doi:10.1162/jocn.2010.21600.

Griffiths, I., Klugmann, M., Anderson, T., Yool, D., Thomson, C., Schwab, M.H., Schneider, A., Zimmermann, F., McCulloch, M., Nadon, N., Nave, K.A. 1998. Axonal swellings and degeneration in mice lacking the major proteolipid of myelin. Science (New York, NY) 280(5369), 1610-3.

Harada, M., Uno, M., Hong, F., Hisaoka, S., Nishitani, H., Matsuda, T. 2002. Diffusion-weighted in vivo localized proton MR spectroscopy of human cerebral ischemia and tumor. NMR Biomed 15(1), 69-74.

Hofling, A.A., Kim, J.H., Fantz, C.R., Sands, M.S., Song, S.K. 2009. Diffusion tensor imaging detects axonal injury and demyelination in the spinal cord and cranial nerves of a murine model of globoid cell leukodystrophy. NMR Biomed 22(10), 1100-6.

Jenkinson, M., Bannister, P., Brady, M., Smith, S. 2002. Improved optimization for the robust and accurate linear registration and motion correction of brain images. Neuroimage 17(2), 82541.

Jenkinson, M., Smith, S. 2001. A global optimisation method for robust affine registration of brain images. Medical image analysis 5(2), 143-56.

Kim, J.H., Budde, M.D., Liang, H.F., Klein, R.S., Russell, J.H., Cross, A.H., Song, S.K. 2006. Detecting axon damage in spinal cord from a mouse model of multiple sclerosis. Neurobiology of disease 21(3), 626-32. doi:10.1016/j.nbd.2005.09.009.

Le Belle, J.E., Harris, N.G., Williams, S.R., Bhakoo, K.K. 2002. A comparison of cell and tissue extraction techniques using high-resolution 1H-NMR spectroscopy. NMR Biomed 15(1), $37-44$.

Liu, Z., Zheng, D., Wang, X., Zhang, J., Xie, S., Xiao, J., Jiang, X. 2011. Apparent diffusion coefficients of metabolites in patients with MELAS using diffusion-weighted MR spectroscopy. AJNR Am J Neuroradiol 32(5), 898-902.

Madden, D.J., Bennett, I.J., Burzynska, A., Potter, G.G., Chen, N.K., Song, A.W. 2012. Diffusion tensor imaging of cerebral white matter integrity in cognitive aging. Biochim Biophys Acta $3,386-400$.

Marner, L., Nyengaard, J.R., Tang, Y., Pakkenberg, B. 2003. Marked loss of myelinated nerve fibers in the human brain with age. J Comp Neurol 462(2), 144-52.

Murray, M.E., Vemuri, P., Preboske, G.M., Murphy, M.C., Schweitzer, K.J., Parisi, J.E., Jack, C.R., Jr., Dickson, D.W. 2012. A quantitative postmortem MRI design sensitive to white matter hyperintensity differences and their relationship with underlying pathology. J Neuropathol Exp Neurol 71(12), 1113-22.

Nicolay, K., Braun, K.P., Graaf, R.A., Dijkhuizen, R.M., Kruiskamp, M.J. 2001. Diffusion NMR spectroscopy. NMR Biomed 14(2), 94-111.

Peters, A. 2002. The effects of normal aging on myelin and nerve fibers: a review. J Neurocytol 31(8-9), 581-93.

Peters, A., Kemper, T. 2012. A review of the structural alterations in the cerebral hemispheres of the aging rhesus monkey. Neurobiol Aging 33(10), 2357-72.

Peters, A., Sethares, C. 2002. Aging and the myelinated fibers in prefrontal cortex and corpus callosum of the monkey. J Comp Neurol 442(3), 277-91.

Pierpaoli, C., Barnett, A., Pajevic, S., Chen, R., Penix, L.R., Virta, A., Basser, P. 2001. Water diffusion changes in Wallerian degeneration and their dependence on white matter 
architecture. Neuroimage 13(6 Pt 1), 1174-85.

Posse, S., Cuenod, C.A., Le Bihan, D. 1993. Human brain: proton diffusion MR spectroscopy. Radiology 188(3), 719-25.

Provencher, S.W. 2001. Automatic quantitation of localized in vivo 1H spectra with LCModel. NMR Biomed 14(4), 260-4.

Reuter-Lorenz, P.A., Stanczak, L. 2000. Differential effects of aging on the functions of the corpus callosum. Dev Neuropsychol 18(1), 113-37.

Ronen, I., Budde, M., Ercan, E., Annese, J., Techawiboonwong, A., Webb, A. 2014. Microstructural organization of axons in the human corpus callosum quantified by diffusionweighted magnetic resonance spectroscopy of $\mathrm{N}$-acetylaspartate and post-mortem histology. Brain Struct Funct 219(5), 1773-85.

Saab, A.S., Tzvetanova, I.D., Nave, K.A. 2013. The role of myelin and oligodendrocytes in axonal energy metabolism. Current opinion in neurobiology 23(6), 1065-72. doi:10.1016/j.conb.2013.09.008.

Salat, D.H., Tuch, D.S., Greve, D.N., van der Kouwe, A.J., Hevelone, N.D., Zaleta, A.K., Rosen, B.R., Fischl, B., Corkin, S., Rosas, H.D., Dale, A.M. 2005. Age-related alterations in white matter microstructure measured by diffusion tensor imaging. Neurobiol Aging 26(8), 121527.

Sandell, J.H., Peters, A. 2003. Disrupted myelin and axon loss in the anterior commissure of the aged rhesus monkey. J Comp Neurol 466(1), 14-30.

Smith, S.M., Jenkinson, M., Johansen-Berg, H., Rueckert, D., Nichols, T.E., Mackay, C.E., Watkins, K.E., Ciccarelli, O., Cader, M.Z., Matthews, P.M., Behrens, T.E. 2006. Tractbased spatial statistics: voxelwise analysis of multi-subject diffusion data. Neuroimage 31(4), 1487-505.

Song, S.K., Sun, S.W., Ramsbottom, M.J., Chang, C., Russell, J., Cross, A.H. 2002. Dysmyelination revealed through MRI as increased radial (but unchanged axial) diffusion of water. Neuroimage 17(3), 1429-36.

Sotiropoulos, S.N., Hernandez-Fernandez, M., Vu, A.T., Andersson, J.L., Moeller, S., Yacoub, E., Lenglet, C., Ugurbil, K., Behrens, T.E., Jbabdi, S. 2016. Fusion in diffusion MRI for improved fibre orientation estimation: An application to the 3T and 7T data of the Human Connectome Project. Neuroimage 134, 396-409.

Sullivan, E.V., Pfefferbaum, A. 2006. Diffusion tensor imaging and aging. Neurosci Biobehav Rev 30(6), 749-61.

Tang, Y., Nyengaard, J.R., Pakkenberg, B., Gundersen, H.J. 1997. Age-induced white matter changes in the human brain: a stereological investigation. Neurobiol Aging 18(6), 609-15.

Teipel, S.J., Meindl, T., Wagner, M., Stieltjes, B., Reuter, S., Hauenstein, K.H., Filippi, M., Ernemann, U., Reiser, M.F., Hampel, H. 2010. Longitudinal changes in fiber tract integrity in healthy aging and mild cognitive impairment: a DTI follow-up study. Journal of Alzheimer's disease : JAD 22(2), 507-22. doi:10.3233/jad-2010-100234.

Urenjak, J., Williams, S.R., Gadian, D.G., Noble, M. 1993. Proton nuclear magnetic resonance spectroscopy unambiguously identifies different neural cell types. The Journal of neuroscience : the official journal of the Society for Neuroscience 13(3), 981-9.

Wallimann, T., Wyss, M., Brdiczka, D., Nicolay, K., Eppenberger, H.M. 1992. Intracellular compartmentation, structure and function of creatine kinase isoenzymes in tissues with high and fluctuating energy demands: the 'phosphocreatine circuit' for cellular energy homeostasis. The Biochemical journal 281 ( Pt 1), 21-40.

Wang, X., Cusick, M.F., Wang, Y., Sun, P., Libbey, J.E., Trinkaus, K., Fujinami, R.S., Song, S.K. 2014. Diffusion basis spectrum imaging detects and distinguishes coexisting subclinical inflammation, demyelination and axonal injury in experimental autoimmune encephalomyelitis mice. NMR Biomed 27(7), 843-52. doi:10.1002/nbm.3129.

Wheeler-Kingshott, C.A., Cercignani, M. 2009. About "axial" and "radial" diffusivities. Magn Reson Med 61(5), 1255-60. 
Winkler, A.M., Ridgway, G.R., Webster, M.A., Smith, S.M., Nichols, T.E. Permutation inference for the general linear model. Neuroimage 2014 92:381-97.(doi), 10.1016/j.neuroimage.2014.01.060. Epub Feb 11.

Wood, E.T., Ercan, A.E., Branzoli, F., Webb, A., Sati, P., Reich, D.S., Ronen, I. 2015. Reproducibility and optimization of in vivo human diffusion-weighted MRS of the corpus callosum at 3 T and 7 T. NMR Biomed 28(8), 976-87. doi:10.1002/nbm.3340.

Wood, E.T., Ronen, I., Techawiboonwong, A., Jones, C.K., Barker, P.B., Calabresi, P., Harrison, D., Reich, D.S. 2012. Investigating axonal damage in multiple sclerosis by diffusion tensor spectroscopy. The Journal of neuroscience : the official journal of the Society for Neuroscience 32(19), 6665-9. doi:10.1523/jneurosci.0044-12.2012.

Wozniak, J.R., Lim, K.O. 2006. Advances in white matter imaging: a review of in vivo magnetic resonance methodologies and their applicability to the study of development and aging. Neurosci Biobehav Rev 30(6), 762-74.

Zheng, D.D., Liu, Z.H., Fang, J., Wang, X.Y., Zhang, J. 2012. The effect of age and cerebral ischemia on diffusion-weighted proton MR spectroscopy of the human brain. AJNR Am J Neuroradiol 33(3), 563-8.

Zhu, X.H., Lu, M., Lee, B.Y., Ugurbil, K., Chen, W. 2015. In vivo NAD assay reveals the intracellular NAD contents and redox state in healthy human brain and their age dependences. Proceedings of the National Academy of Sciences of the United States of America 112(9), 2876-81. doi:10.1073/pnas.1417921112. 